\title{
Allogeneic stem cell transplantation in a blast-phase chronic myeloid leukemia patient with carbapenem-resistant Klebsiella pneumoniae tricuspid valve endocarditis: A case report
}

\author{
BULENT KANTARCIOGLU ${ }^{1}$, HUSEYIN SAFFET BEKOZ ${ }^{1}$, FATIH ERKAM OLGUN ${ }^{2}$, \\ BEYTULLAH CAKAL ${ }^{2}$, BURAK ARKAN ${ }^{3}$, HALIL TURKOGLU ${ }^{3}$, ALI MERT ${ }^{4}$ and DENIZ SARGIN ${ }^{1}$ \\ ${ }^{1}$ Division of Hematology, Department of Internal Medicine; Departments of ${ }^{2}$ Cardiology, ${ }^{3}$ Cardiovascular Surgery \\ and ${ }^{4}$ Infectious Diseases and Clinical Microbiology, Istanbul Medipol University, 34214 Istanbul, Turkey
}

Received May 9, 2016; Accepted July 15, 2016

DOI: $10.3892 / \mathrm{mco} .2016 .995$

\begin{abstract}
In chronic myeloid leukemia (CML), the occurrence of blastic transformation is rare. Treatment outcome is generally poor. Allogeneic stem cell transplantation (allo-SCT) is the only potentially curative treatment option for advanced-phase CML. Infections caused by carbapenem-resistant Klebsiella pneumoniae (CRKP) isolates are associated with high morbidity and mortality rates, particularly in patients with haematological malignancies. Infection and colonization by these multiresistant bacteria may represent a challenge in SCT recipients for the management of post-transplantation complications, as well as for the eligibility to receive a transplant in patients who acquire the pathogen prior to the procedure. We herein report the case of a blast-phase CML patient with a highly resistant, CRKP-associated tricuspid valve endocarditis, who was treated with a combination of systemic antimicrobial therapy and surgical valve repair, and subsequently underwent a successful allo-SCT.
\end{abstract}

\section{Introduction}

Infections caused by carbapenem-resistant Klebsiella pneumoniae (CRKP) represent a significant public health concern worldwide. Infections caused by CRKP isolates are associated with high morbidity and mortality rates, particularly in patients with haematological malignancies. Infection and colonization by these multiresistant bacteria may represent a challenge in SCT recipients for the management of post-transplantation complications, as well as for the eligibility

Correspondence to: Dr Bulent Kantarcioglu, Division of Hematology, Department of Internal Medicine, Istanbul Medipol University, TEM Avrupa Otoyolu Goztepe Cikisi 1, 34214 Istanbul, Turkey

E-mail: bulentkantarcioglu@gmail.com

Key words: allogeneic stem cell transplantation, infective endocarditis, carbapenem-resistant Klebsiella pneumoniae to receive a transplant in patients who acquire the pathogen prior to the procedure $(1,2)$.

We herein report the case of a blast-phase chronic myeloid leukemia (BP-CML) patient with a highly resistant, CRKP-associated tricuspid valve endocarditis, who was treated with a combination of systemic antimicrobial therapy and surgical valve repair, and subsequently underwent successful allogeneic stem cell transplantation (allo-SCT). To the best of our knowledge, this is the first reported CRKP tricuspid valve endocarditis in the medical literature.

\section{Case report}

A 50-year-old female patient with BP-CML was referred to the Istanbul Medipol University in January 2015. The patient had been diagnosed with CML in Libya (July, 2014). The disease was in chronic phase, so treatment with imatinib was started by her physicians. After treatment with imatinib for 3 months, the patient developed progressive disease with myeloid blastic transformation and $7+3$ induction chemotherapy was initiated. During this treatment, the patient was intubated for severe pneumonia with sepsis and had to stay in the intensive care unit (ICU) for $\sim 2$ months in a Libyan hospital. The bone marrow biopsy that was performed after the infection had resolved was consistent with complete remission of the leukaemia; thus, the patient was referred to our hospital for an allo-SCT.

On admittance to the hospital, the patient was febrile. Peripheral and central venous port blood cultures were collected, which grew KP that was susceptible only to tigecycline. As venous port infection was suspected, the patient's venous port was removed and she was treated with tigecycline and meropenem (Table I, 1st bacteraemia). Treatment was discontinued after 14 days. The patient was in good condition and preparations for allo-SCT were initiated. During her work-up, the patient developed new onset fever 5 days after the cessasion of antibiotics. The blood cultures again revealed KP, with the same resistance pattern (Table I, 2nd bacteraemia). Tigecycline and imipenem were administered. The fever resolved and the acute-phase parameters returned to normal levels within 5 days. Treatment was again discontinued after 2 weeks, but the patient developed fever shortly after. 
Table I. Antibiotic resistance pattern of bacteraemia and endocarditis.

\begin{tabular}{|c|c|c|c|c|c|c|c|c|}
\hline \multirow{2}{*}{$\begin{array}{l}\text { Antibiotics } \\
\text { Ampicillin-sulbactam }\end{array}$} & \multicolumn{2}{|c|}{ 1st bacteraemia } & \multicolumn{2}{|c|}{ 2nd bacteraemia } & \multicolumn{2}{|c|}{ 3rd bacteraemia } & \multicolumn{2}{|c|}{$\begin{array}{l}\text { Culture of } \\
\text { vegetation }\end{array}$} \\
\hline & $>32$ & $\mathrm{R}$ & $>32$ & $\mathrm{R}$ & $>32$ & $\mathrm{R}$ & $>32$ & $\mathrm{R}$ \\
\hline Piperacillin & $>128$ & $\mathrm{R}$ & $>128$ & $\mathrm{R}$ & $>128$ & $\mathrm{R}$ & $>128$ & $\mathrm{R}$ \\
\hline Piperacillin-tazobactam & $>128$ & $\mathrm{R}$ & $>128$ & $\mathrm{R}$ & $>128$ & $\mathrm{R}$ & $>128$ & $\mathrm{R}$ \\
\hline Ceftazidime & 16 & $\mathrm{R}$ & 4 & I & 16 & $\mathrm{R}$ & 4 & $\mathrm{R}$ \\
\hline Cefoperazone-sulbactam & $>64$ & $\mathrm{R}$ & $>64$ & $\mathrm{R}$ & $>64$ & $\mathrm{R}$ & $>64$ & $\mathrm{R}$ \\
\hline Cefepime & $>64$ & $\mathrm{R}$ & $>64$ & $\mathrm{R}$ & $>64$ & $\mathrm{R}$ & $>64$ & $\mathrm{R}$ \\
\hline Imipenem & $>16$ & $\mathbf{R}$ & 2 & I & 8 & $\mathbf{R}$ & $>16$ & $\mathbf{R}$ \\
\hline Meropenem & $>16$ & $\mathbf{R}$ & $>16$ & $\mathbf{R}$ & $>16$ & $\mathbf{R}$ & $>16$ & $\mathbf{R}$ \\
\hline Amikacin & $>64$ & $\mathrm{R}$ & $>64$ & $\mathrm{R}$ & $>64$ & $\mathrm{R}$ & $>64$ & $\mathrm{R}$ \\
\hline Gentamicin & $>16$ & $\mathbf{R}$ & $>16$ & $\mathbf{R}$ & $>16$ & $\mathbf{R}$ & $>16$ & $\mathbf{R}$ \\
\hline Netilmicin & 8 & I & 8 & I & 8 & I & 8 & I \\
\hline Ciprofloxacin & $>4$ & $\mathrm{R}$ & $>4$ & $\mathrm{R}$ & $>4$ & $\mathrm{R}$ & $>4$ & $\mathrm{R}$ \\
\hline Levofloxacin & $>8$ & $\mathrm{R}$ & $>8$ & $\mathrm{R}$ & $>8$ & $\mathrm{R}$ & $>8$ & $\mathrm{R}$ \\
\hline Tetracycline & 4 & I & 4 & I & 8 & I & 8 & I \\
\hline Tigecycline & 2 & $\mathbf{S}$ & 1 & $\mathbf{S}$ & 4 & $\mathbf{I}$ & 4 & $\mathbf{I}$ \\
\hline Colistin & $>16$ & $\mathbf{R}$ & $\mathbf{R}$ & $\mathbf{R}$ & $>16$ & $\mathbf{R}$ & $>16$ & $\mathbf{R}$ \\
\hline Trimetoprim-sulfamethoxazole & $>320$ & $\mathrm{R}$ & $>320$ & $\mathrm{R}$ & $>320$ & $\mathrm{R}$ & $>320$ & $\mathrm{R}$ \\
\hline
\end{tabular}

Bold print indicates common antibiotics used in the treatment of CRKP infections. S, susceptible; I, intermediate; R, resistant.

Repeated blood cultures again grew pan-resistant KP (Table I, 3rd bacteraemia). Rectal swabs that were collected during the hospitalisation period were all negative for the carrier state of CRKP. Transthoracic echocardiogram was performed for repeated bacteraemia, and revealed enlarged right cardiac chambers, moderate tricuspid valve insufficiency and a mass sized $1.5 \times 1.4 \mathrm{~cm}$ on the tricuspid valve, compatible with a septic vegetation. With the diagnosis of tricuspid valve endocarditis due to a multiresistant pathogen, the patient was operated for excision of the vegetation and tricuspid valve repair (Fig. 1). The patient was treated with imipenem-cilastatin $1 \mathrm{~g}$ intravenously every $6 \mathrm{~h}$, plus gentamicin $80 \mathrm{mg}$ intravenously every $8 \mathrm{~h}$, plus sulbactam $1 \mathrm{~g}$ intravenously every $6 \mathrm{~h}$ for 4 weeks.

During the treatment period of endocarditis, the patient was also treated for CML with nilotinib. However, polymerase chain reaction (PCR) for Bcr/Abl remained positive, indicating residual disease. After the infection had resolved, unmanipulated peripheral blood allo-SCT from a full-matched sibling donor was performed in November, 2015. The reduced intensity conditioning (RIC) regimen included fludarabine at $30 \mathrm{mg} / \mathrm{m}^{2} /$ day for 5 days and busulfan at $3.2 \mathrm{mg} / \mathrm{kg} /$ day for 2 days. Cyclosporin A, short-course methotrexate and antithymocyte globulin were used for acute graft-vs.-host disease (GVHD) prophylaxis. Allo-SCT was performed without complications; neutrophil engraftment was achieved on day +15 and thrombocyte engraftment was achieved on day +14 post-transplantation. The patient is currently in complete remission with complete chimerism, without evidence of any GVHD, for $>3$ months after allo-SCT. The PCR of Bcr/Abl is also negative, showing no signs of residual disease.

Written informed consent was obtained from the patient for the publication of the case details.

\section{Discussion}

KP is one of the most important causes of health care- and ICU-acquired infections (3) and has been reported as the second overall cause of Gram-negative bloodstream infections (BSI) after Escherichia coli. However, Klebsiella species are a particularly rare cause $(1.5 \%)$ of Gram-negative bacterial endocarditis. Klebsiella species cause $1.2 \%$ of the cases of native valve endocarditis and $4.1 \%$ of the cases of prosthetic valve endocarditis. It has been reported that the most commonly affected valve is the aortic valve, followed by the mitral and tricuspid valves. The most common source of bacteraemia is defined as urinary tract infection and infection of a pacemaker $(4,5)$. In our patient, the source was an infected port catheter, which led to tricuspid valve endocarditis. To the best of our knowledge, this is the first reported case of tricuspid valve endocarditis due to CRKP.

$\mathrm{KP}$ is a rare but extremely dangerous cause of bacterial endocarditis. Large vegetations, valve perforation and root abscesses are frequent complications. Combined medical and surgical treatment is usually required. The reported mortality rate of Klebsiella endocarditis is $49 \%$ (6). The main predictors of CRKP infection are poor functional status, high severity of underlying conditions, ICU stay and prior exposure to antimicrobial agents. Patients with haematological malignancies and haematopoietic stem cell transplant recipients are particularly vulnerable to CRKP infections due to chemotherapy-induced gastrointestinal mucositis, prolonged hospitalizations and neutropenia, as well as the frequent use of broad-spectrum antibacterial agents (7). Our patient combined several risk factors, namely poor functional status, serious haematological malignancy, history of active chemotherapy, prolonged 

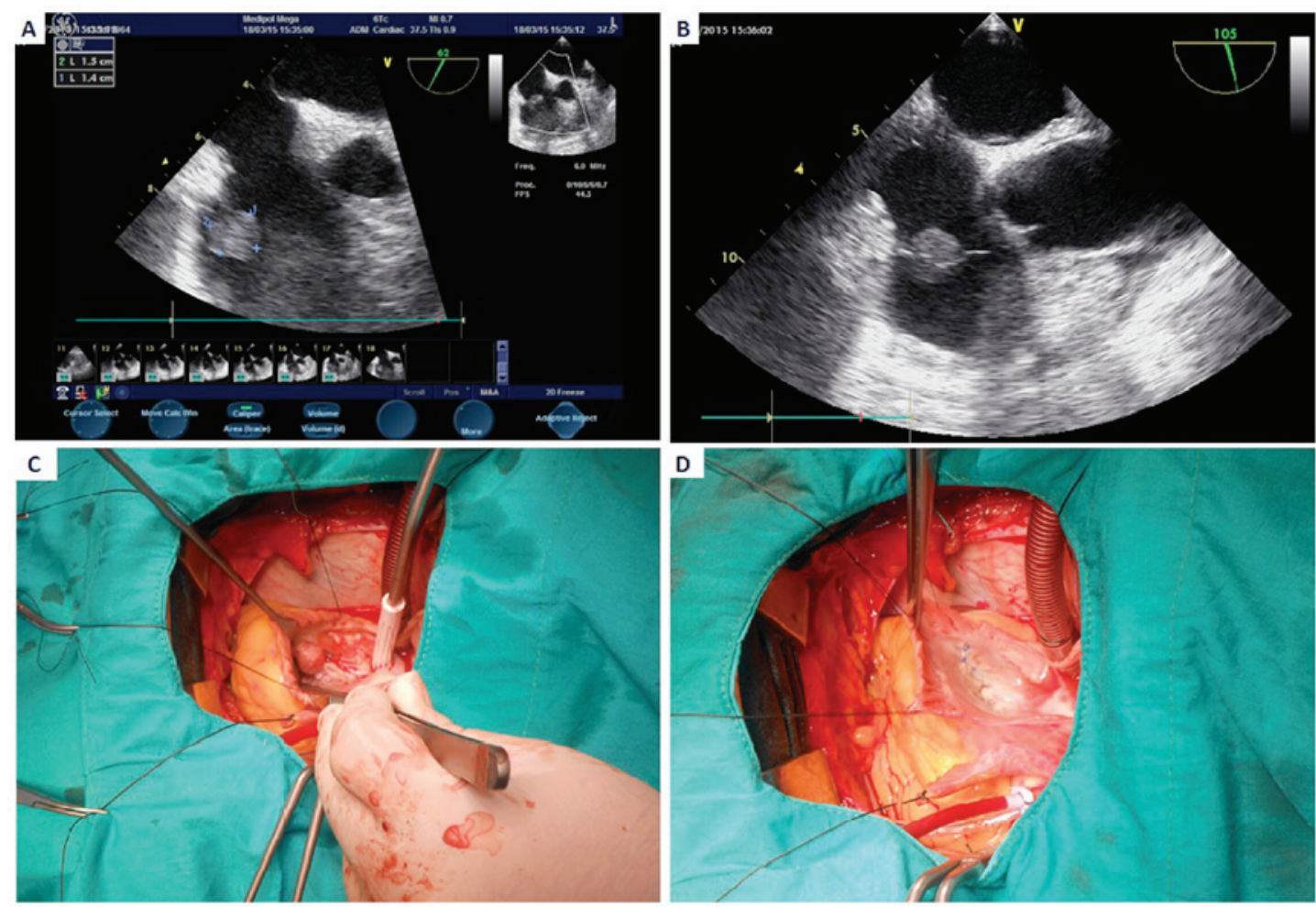

Figure 1. (A and B) Echocardiographic images of the patient, showing a vegetation on the tricuspid valve. Intraoperative images of the (C) tricuspid valve with the vegetation and (D) excision of the vegetation and valve repair.

hospitalization with ICU stay and the use of broad-spectrum antibiotics, making her treatment more challenging.

There is currently no consensus regarding the optimal regimen for treating CRKP. Treatment selection is generally based on the results of retrospective and/or single-center studies of CRKP BSIs. It is currently widely accepted that the combination of a first-line antibiotic, mainly carbapenems, at higher doses to overcome resistance, and a second-line antibiotic with Gram-negative activity for which resistance has hopefully not yet developed (for example, colistin, tigecycline, gentamicin, fosfomycin) is the most suitable treatment option for CRKP BSIs $(4,8)$. Our patient was successfully treated with a combination of high-dose imipenem-cilastatin, gentamicin and sulbactam.

In tricuspid valve endocarditis, surgery is indicated for persistent right-sided heart failure despite medical therapy, recurrent pulmonary septic emboli, septic shock, abscess formation or failure of antimicrobial therapy to control the infection (7). In our patient, recurrent bacteraemia with a multiresistant pathogen was the main indication for valve surgery. The surgical options comprise tricuspid valve reconstruction and tricuspid valve replacement. In tricuspid valve endocarditis, both options are applicable. However, the main limitation of tricuspid valve replacement was the need for long-term anticoagulation, which would be a major problem for performing heart surgery, as well as for performing allo-SCT in our patient. In case of the latter condition, data are limited, as there is only one reported case of allo-SCT being performed in a patient with a prosthetic valve (9). Another problem is the increased risk of bleeding, infectious complications and reoperation rates of patients with haematological malignancies undergoing cardiac surgery (10). In our case, no cardiac complications occurred, without the need for additional measures during allo-SCT.

In CML, the incidence of BP is a rare condition. Optimal outcomes are achieved with allo-SCT after obtaining complete remission with induction chemotherapy (11). In our patient, complete remission after induction chemotherapy was an absolute indication for allo-SCT. However, recent CRKP endocarditis was a challenge when deciding to perform allo-HSCT. Satlin et al published a retrospective analysis of 18 malignant haematological patients with carbapenem-resistant Enterobacteriaceae BSI. In that study, initial empirical antimicrobial therapy was active in only $11 \%$ of the patients and the mortality rate was $56 \%$ (12). In another multicenter retrospective study, Girmenia et al assessed the epidemiology and prognostic factors of CRKP infections in an SCT setting. CRKP infections were reported in $53.4 \%$ of the centers and were documented in $0.4 \%$ of autologous and $2 \%$ of allogeneic SCTs. CRKP colonization was followed by an infection in $\sim 30 \%$ of the cases. The infection-related mortality rate was $16 \%$ in autologous and $64.4 \%$ in allogeneic SCT. A pre-transplant CRKP infection and an inadequate first-line treatment were associated with an increased mortality in allo-SCT patients who developed a CRKP infection. Indeed, despite the administration of first-line CRKP-targeted antibiotic therapy (CTAT), 55\% of the patients who received CTAT succumbed to the disease (13). Zuckerman et al evaluated the eradication of CRKP carrier state in 15 patients undergoing intensive chemotherapy or SCT. In that study, the eradication rate was $66 \%$ and all the patients in whom eradication failed succumbed to the disease, usually due to active CRKP infection (14). According to the results of Kuruvilla et al, recurrence of endocarditis during allo-SCT was the worst outcome, exhibiting a 
very high mortality rate (15). For these reasons, we decided to reduce the transplantation-associated risks with complete eradication of the endocarditis and the CRKP infection prior to the transplantation, and opted for performing a less invasive surgery rather than a mechanical tricuspid valve, which would require a higher level of anticoagulation. We also planned to use an RIC regimen instead of myeloablative conditioning during the transplantation.

In conclusion, we herein report a case of a BP-CML patient with a highly resistant, CRKP-associated tricuspid valve endocarditis, who was treated with a combination of systemic antimicrobial therapy and surgical valve repair, and subsequently underwent successful allo-SCT. To the best of our knowledge, this is the first reported CRKP tricuspid valve endocarditis and the first reported case of CRKP endocarditis that proceeded to undergo a successful allo-SCT in the medical literature. This report demonstrated that extensively resistant CRKP endocarditis may be treated with combination therapy without mechanical heart valve replacement in patients with haematological malignancies. Our case also emphasises the significance of a collaborative multidisciplinary approach in such patients.

\section{References}

1. Doi Y and Paterson DL: Carbapenemase-producing Enterobacteriaceae. Semin Respir Crit Care Med 36: 74-84, 2015.

2. Girmenia C, Viscoli C, Piciocchi A, Cudillo L, Botti S, Errico A, Sarmati L, Ciceri F, Locatelli F, Giannella M, et al: Management of carbapenem resistant Klebsiella pneumoniae infections in stem cell transplant recipients: An Italian multidisciplinary consensus statement. Haematologica 100: e373-e376, 2015.

3. Vardakas KZ, Matthaiou DK, Falagas ME, Antypa E, Koteli A and Antoniadou E: Characteristics, risk factors and outcomes of carbapenem-resistant Klebsiella pneumoniae infections in the intensive care unit. J Infect 70: 592-599, 2015.

4. Girometti N, Lewis RE, Giannella M, Ambretti S, Bartoletti M, Tedeschi S, Tumietto F, Cristini F, Trapani F, Gaibani P and Viale P: Klebsiella pneumoniae bloodstream infection: Epidemiology and impact of inappropriate empirical therapy. Medicine (Baltimore) 93: 298-309, 2014.
5. Anderson MJ and Janoff EN: Klebsiella endocarditis: Report of two cases and review. Clin Infect Dis 26: 468-474, 1998.

6. Srinivas KH, Sharma R, Agrawal N and Manjunath CN: Silent destruction of aortic and mitral valve by Klebsiella pneumoniae endocarditis. BMJ Case Rep 2013: pii: bcr2013200704, 2013.

7. Yong MS, Coffey S, Prendergast BD, Marasco SF, Zimmet AD, McGiffin DC and Saxena P: Surgical management of tricuspid valve endocarditis in the current era: A review. Int J Cardiol 202: 44-48, 2016.

8. Satlin MJ, Jenkins SG and Walsh TJ: The global challenge of carbapenem-resistant Enterobacteriaceae in transplant recipients and patients with hematologic malignancies. Clin Infect Dis 58: 1274-1283, 2014

9. Prébet T, Devillier R, Fürst S, Vey N and Blaise D: Allogeneic hematopoietic SCT and mechanical heart valve: Feasibility of reduced toxicity myeloablative conditioning. Bone Marrow Transplant 45: 1574-1575, 2010.

10. Fecher AM, Birdas TJ, Haybron D, Papasavas PK, Evers D and Caushaj PF: Cardiac operations in patients with hematologic malignancies. Eur J Cardiothorac Surg 25: 537-540, 2004.

11. Saußele S and Silver RT: Management of chronic myeloid leukemia in blast crisis. Ann Hematol 94 (Suppl 2): S159-S165, 2015.

12. Satlin MJ, Calfee DP, Chen L, Fauntleroy KA, Wilson SJ, Jenkins SG, Feldman EJ, Roboz GJ, Shore TB, Helfgott DC, et al: Emergence of carbapenem-resistant Enterobacteriaceae as causes of bloodstream infections in patients with hematologic malignancies. Leuk Lymphoma 54: 799-806, 2013.

13. Girmenia C, Rossolini GM, Piciocchi A, Bertaina A, Pisapia G, Pastore D, Sica S, Severino A, Cudillo L, Ciceri F, et al: Infections by carbapenem-resistant Klebsiella pneumoniae in SCT recipients: A nationwide retrospective survey from Italy. Bone Marrow Transplant 50: 282-288, 2015.

14. Zuckerman T, Benyamini N, Sprecher H, Fineman R, Finkelstein R, Rowe JM and Oren I: SCT in patients with carbapenem resistant Klebsiella pneumoniae: A single center experience with oral gentamicin for the eradication of carrier state. Bone Marrow Transplant 46: 1226-1230, 2011.

15. Kuruvilla J, Forrest DL, Lavoie JC, Nantel SH, Shepherd JD, Song KW, Sutherland HJ, Toze CL, Hogge DE and Nevill TJ: Characteristics and outcome of patients developing endocarditis following hematopoietic stem cell transplantation. Bone Marrow Transplant 34: 969-973, 2004. 